\title{
Heterogeneities in Fullerene Nanoparticle Aggregates Affecting Reactivity, Bioactivity, and Transport
}

\author{
So-Ryong Chae, ${ }^{t, \pm, s}$ Appala R. Badireddy, ${ }^{t, \neq, \S}$ Jeffrey Farner Budarz, ${ }^{t, \neq}$ Shihong Lin, ${ }^{t, \pm}$ Yao Xiao, ${ }^{t, \neq}$ \\ Mathieu Therezien, ${ }^{t, \neq}$ and Mark R. Wiesner ${ }^{t, \pm, *}$ \\ ${ }^{\dagger}$ Department of Civil and Environmental Engineering, Pratt School of Engineering and ${ }^{\ddagger}$ Center for the Environmental Implications of NanoTechnology (CEINT), Duke

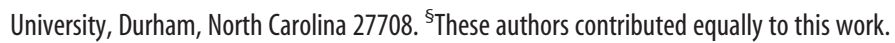

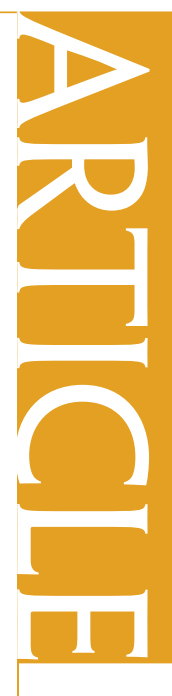

$\mathrm{T}$ he reactivity of $\mathrm{C}_{60}$ fullerene nanoparticles is influenced by the size and aggregation state of these particles. ${ }^{1,2}$ Environmental media are likely to influence the degree of aggregation ${ }^{3}$ with potential implications that extend to the bioavailability and toxicity of these materials. The ever-increasing use of fullerenes in commercial products and applications has raised concerns over the potential consequence of environmental and human exposure. ${ }^{3-5}$ These concerns stem from the increasing use of $C_{60}$, their inevitable release into the water bodies, and their photosensitivity which is responsible for the production of reactive oxygen species (ROS) such as singlet oxygen $\left({ }^{1} \mathrm{O}_{2}\right)$ and electron-donor-mediated superoxide $\left(\mathrm{O}_{2}{ }^{--}\right)$, which in turn could be a precursor to Fenton-like reactions in the presence of transient metals (e.g., $\mathrm{Fe}^{3+}$ ), leading to the formation of hydroxyl radicals, and thereby induce in vitro and (or) in vivo oxidative stress. ${ }^{6-8}{ }^{1} \mathrm{O}_{2}$ and $\mathrm{O}_{2}{ }^{\bullet-}$ have been shown to oxidize organic compounds, ${ }^{9-11}$ inactivate viruses, and indiscriminately kill cells by means of compromising their membrane integrity, damaging DNA and other internal organelles, and disrupting cell signaling pathways. ${ }^{12-15}$

With a reported solubility of $1.3 \times 10^{-11}$ $\mu \mathrm{g} / \mathrm{L}$ in water, individual molecules of $\mathrm{C}_{60}$ are not typically present in water. ${ }^{16}$ Rather, $\mathrm{C}_{60}$ tends to form aggregates (referred to as $n C_{60}$, where $n$ is the number of single $C_{60}$ cages in an aggregate). Depending on the mode of preparation, ${ }^{17}$ the structure of these aggregates may range from crystalline to fractal. Thus, in addition to differences attributable to residual impurities introduced in the preparation of $n \mathrm{C}_{60}{ }^{18}$ the mode of $n C_{60}$ preparation may yield differ-

ABSTRACT Properties of nanomaterial suspensions are typically summarized by average values for the purposes of characterizing these materials and interpreting experimental results. We show in this work that the heterogeneity in aqueous suspensions of fullerene $C_{60}$ aggregates $\left(n C_{60}\right)$ must be taken into account for the purposes of predicting nanomaterial transport, exposure, and biological activity. The production of reactive oxygen species (ROS), microbial inactivation, and the mobility of the aggregates of the $n C_{60}$ in a silicate porous medium all increased as suspensions were fractionated to enrich with smaller aggregates by progressive membrane filtration. These size-dependent differences are attributed to an increasing degree of hydroxylation of $n \mathrm{C}_{60}$ aggregates with decreasing size. As the quantity and influence of these more reactive fractions may increase with time, experiments evaluating fullerene transport and toxicity end points must take into account the evolution and heterogeneity of fullerene suspensions.

KEYWORDS: nanomaterial · heterogeneity $\cdot$ fullerene $C_{60} \cdot$ reactive oxygen species (ROS) $\cdot$ microbial inactivation $\cdot$ transport $\cdot$ size-dependent differences

ent physical characteristics of $n \mathrm{C}_{60}$ suspensions. Procedures, including sonication, that are employed to disperse or break up initial fullerene powder for preparation of stable suspensions have been observed to modify the surface chemistry of $C_{60}$. Labille et al. have shown Fourier transform infrared (FT-IR) absorption at wavelengths consistent with $\mathrm{C}-\mathrm{O}$ stretching after sonication, pointing to the hydroxylation of fullerene due to the suspension mechanism. ${ }^{19}$ Structural and chemical differences in $n \mathrm{C}_{60}$ translate to differences in reactivity. Colloidal aggregates $\left(n C_{60}\right)$ of fullerene in water $\left(\mathrm{aqu} / \mathrm{nC}_{60}\right)$ have been reported to produce little to no detectable ROS, $9,20,21$ despite having a quantum yield near unity, whereas polyhydroxylated fullerene (fullerol, $\left.\left(\mathrm{C}_{60}(\mathrm{OH})_{24}\right)\right)_{1}{ }^{22}$ a known ROS generator in aqueous suspensions, ${ }^{9,10,23}$ possesses a quantum yield that is nearly an order of magnitude smaller. It is reasonable to conclude that varying conditions of sonication will also produce varying degrees of hydroxylated $\mathrm{C}_{60}$ with properties approaching those of fullerol.

\section{*Address correspondence to wiesner@duke.edu.}

Received for review March 26, 2010 and accepted July 29, 2010.

Published online August 13, 2010. $10.1021 / \mathrm{nn} 100620 \mathrm{~d}$

๑) 2010 American Chemical Society 


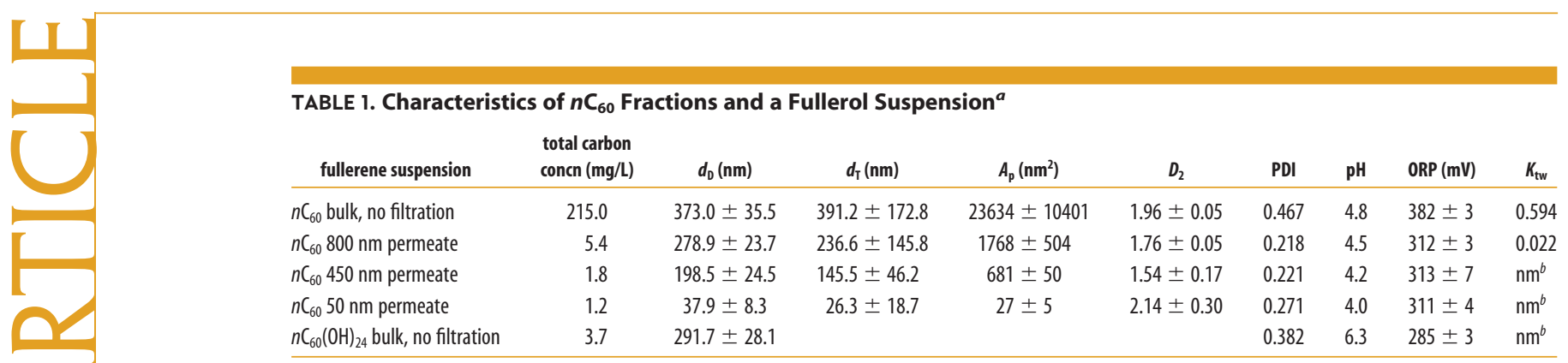

${ }^{a}$ All values are means $\pm 95 \%$ confidence interval $(n=3)$ with the exception of image analysis results (number of aggregates $\left.=100\right) ; d_{0}$ is hydrodynamic diameter of fullerene aggregates measured by DLS $(n=3) ; d_{\mathrm{T}}$ is the mean diameter of a random sample of 100 aggregates from the TEM images; $A_{\mathrm{p}}$ is the projected area; $D_{2}$ is the twodimensional fractal dimension determined from TEM images of aggregates $(n=100)$; PDI is polydispersivity index of the aggregates; ORP is oxidation -reduction potential of the suspensions; and $K_{\mathrm{tw}}$ is toluene/water partition coefficient of the aggregates. ${ }^{b}$ Not measurable: the concentration of $\mathrm{C}_{60}$ in toluene was below detection limit by HPLC (i.e., $15 \mu \mathrm{g} / \mathrm{L})$.

Variations in suspension characteristics and reactivity such as these may be responsible in part for the variety of biological responses reported in the literature to date. For example, inhibition of both Gram-negative and Gram-positive bacteria via respiratory chain arrest and growth inhibition attributed to the production of ROS has been reported, ${ }^{24,25}$ while other studies reported that $n C_{60}$ itself exerts bacterial inhibition independent of ROS production. ${ }^{26}$ These differences in the literature are augmented by anecdotal reports of variability in $C_{60}$ toxicity as a function of the age or storage conditions of stock suspensions.

However, variability between suspensions is compounded by the fact that a given suspension of $n C_{60}$ is itself heterogeneous, known to consist of aggregates of varying size and shapes. The current work characterizes this heterogeneity, documents the wide range of chemical and biological activity of different fractions of $n \mathrm{C}_{60}$, and underscores the difficulty that this variability imposes in interpreting data on fullerene transport and bioactivity. We explore differences in size, surface chemistry, and structural density of $n C_{60}$ aggregates fractionated through membrane filtration and the associated differences in ROS production and subsequent impacts on microbial receptors.

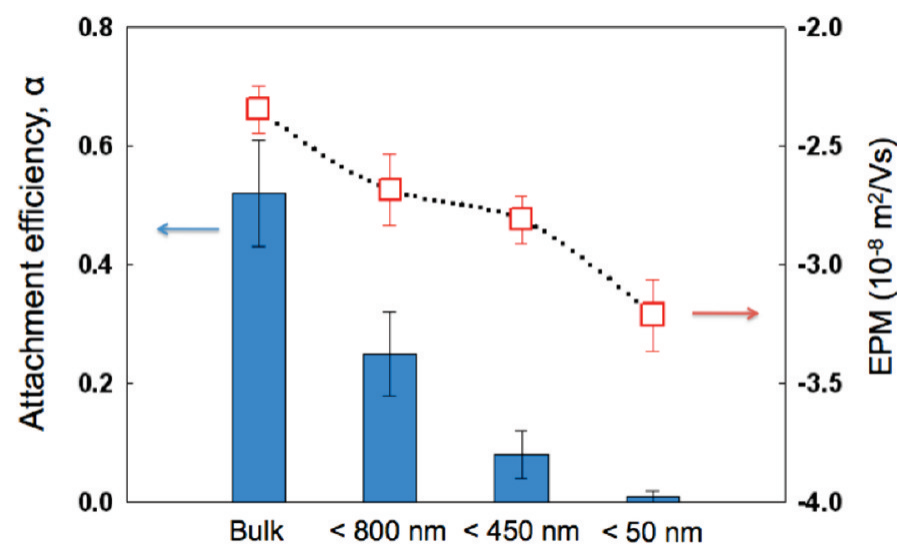

Figure 1. Attachment efficiency of fullerene aggregates onto silica surfaces and electrophoretic mobility (EPM) of fullerene aggregates in water. All values are means $\pm 95 \%$ confidence interval $(n=3)$.

\section{RESULTS AND DISCUSSION}

Characteristics of the Fullerene Suspensions. Characteristics of the suspensions including hydrodynamic diameter $\left(d_{\mathrm{D}}\right)$ as measured by dynamic light scattering (DLS), polydispersivity index (PDI), total carbon (TC) concentration, and the toluene/water partition coefficient $\left(K_{\mathrm{tw}}\right)$ are summarized in Table 1 and Figure 1. Fractionation of aggregate suspensions was performed by using membrane filtration. In general, the physicochemical properties of the initial bulk suspension were not representative of the individual fractions. Figure S1 (Supporting Information) shows the particle size distributions of various size fractions used in this study. Oxidation-reduction potential (ORP) and $\mathrm{pH}$ of the suspensions decreased with sequentially smaller fractions produced by membrane filtration while the absolute value of electrophoretic mobility (EPM) increased. A higher EPM is consistent with a higher degree of hydroxylation and lower affinity for an organic phase such as toluene. It is therefore useful to compare the properties of the $n \mathrm{C}_{60}$ fractions with those of fullerol aggregates, which were smaller and more hydrophilic (as quantified by $K_{\mathrm{tw}}$ ) than those of the bulk $n \mathrm{C}_{60}$ suspension. A greater degree of hydroxylation and a corresponding increase in surface charge (as evidenced by EPM) also suggest that greater charge stabilization should reduce the attachment efficiency of these particles to a negatively charged collector in a porous medium (silica beads) based on the classical Derjaguin-Landau-Verwey-Overbeek (DLVO) theory. ${ }^{27,28}$ Indeed, the affinity between the collector surface and the aggregates, as represented by the attachment efficiency, decreased with aggregate size (Figure 1). However, the difference in the EPMs alone would not suffice to explain the difference in $\alpha$ by simply reasoning that an increase in surface potential (which is reflected in EPM) would increase the electric double layer (EDL) interaction and thus lead to higher stability. After all, the zeta potentials of all four species are fairly high (from $30.5 \mathrm{mV}$ to $41 \mathrm{mV}$ as calculated from the EPM). As the zeta potential of the glass bead surface is also higher than $30 \mathrm{mV}$, 


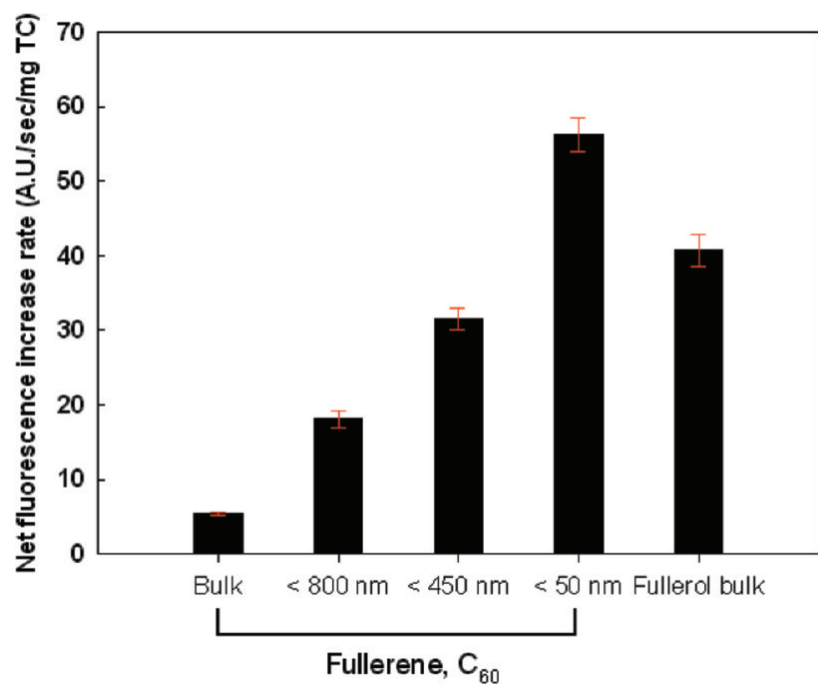

Figure 2. Singlet oxygen production of various fullerene suspensions ( $\mathrm{TC}=1 \mathrm{mg} / \mathrm{L}$ ). All values are means $\pm 95 \%$ confidence interval $(n=3)$.

relatively high attachment efficiencies such as that observed in the 'Bulk' species would not be expected from a simple DLVO model. In addition, assuming all the species were of the same surface potential, the classical DLVO theory predicts that higher attachment efficiency for the smaller particles due to decreased EDL interaction, which is not observed in the data. Such evidence would further support the argument that DLVO theory alone would not successfully explain the results here. We believe that it is the difference in hydrophilicity/hydrophobicity between aggregates of different sizes that contribute significantly to the difference in their affinities towards the surface. The smaller particles/aggregates were more hydrophilic than their larger counterparts and thus had a higher affinity toward the water phase than toward the solid phase as compared to that of the larger particles, which is reflected by the lower attachment efficiency of the smaller fractionated particles/aggregates. Such an increase in stability for the more hydrophilic particles may also be explained by the additional repulsive 'hydration' or 'structural' forces the origin of which is still unclear.

The structural properties of fullerene aggregates measured by transmission electron microscopy (TEM) are summarized in Table 1 and shown in Figure S2 (Supporting Information). The mean diameters as calculated from TEM image analysis $\left(d_{T}\right)$ were statistically indistinguishable from diameters obtained by DLS and are also consistent with trends in projected areas of aggregates $\left(A_{\mathrm{p}}\right)$. As shown in Figure $\mathrm{S} 2$, the fractal dimension of aggregates projected in two-dimension space $\left(D_{2}\right)$ in the bulk $n C_{60}$ corresponds to a more dense arrangement (higher two-dimensional fractal dimension, $D_{2}$ ) than that calculated from fractionated aggregates.
Limits in TEM resolution may be the source of the higher fractal dimension calculated for the smallest size fraction (i.e., $n C_{60}<50 \mathrm{~nm}$ ).

Reactive 0xygen Species Production of the Fullerene Suspensions. Superoxide production in the suspensions under ultraviolet (UV) irradiation was negligible in suspensions as measured by the reduction of XTT (2,3-bis(2-methoxy-4nitro-5-sulfophenyl)-2H-tetrazolium-5carboxanilide). This was anticipated due to the lack of an electron donor required to produce the intermediate $\mathrm{C}_{60}$ radical. However, singlet oxygen production was detected in all samples as measured by fluorescence of SOSG (singlet oxygen sensor green) (Figure 2). In agreement with previous reports, ${ }^{9,29}$ the net increase in fluorescence (calculated as the difference of the observed value from the suspensions and that observed in ultrapure water and normalized per mass of carbon present) was very low in the bulk suspension without membrane filtration by this method. However, as the size of aggregates decreases, the fraction of $\mathrm{C}_{60}$ on the surface of the aggregates increases, and in at least the $<800 \mathrm{~nm}$ and $<450 \mathrm{~nm}$ fractions, the density of aggregates as expressed by $D_{2}$ decreases. These factors lead to a decrease in triplet-triplet annihilation and quenching by ground state $C_{60}$ in the aggregate that allow for greater singlet oxygen production. For similar reasons, ${ }^{9,20}$ singlet oxygen generation of fullerol aggregates was also much higher than that of the bulk $n \mathrm{C}_{60}$. The similarities in the ROS production by the smaller aggregates and that of the fullerol suspension, combined with the lower attachment efficiencies of the smaller fractions (Figure 1), higher EPM (i.e., ל-potentials), and structural data (Table 1), all point to a greater degree of hydroxylation per $C_{60}$ as fractionation proceeds to the smaller sizes (Table 2 shows $p$ values for this hypothesis). As a result, singlet oxygen production in the smallest fraction $(<50$ $\mathrm{nm}$ ) was approximately 10 times greater than that of the bulk $n C_{60}$ suspension. However, ROS production of the smallest fraction was even higher than that of the fullerol suspension $\left(d_{D}=291.7 \mathrm{~nm}\right)$. This latter observation may reflect an optimum level of

TABLE 2. Relationship between Size of Fullerene

Aggregates and Transport through Porous Media and ROS Production (All Values Are Means $\pm 95 \%$ Confidence Interval)

\begin{tabular}{llc}
\multicolumn{1}{c}{ nanoparticle } & attachment efficiency, $\boldsymbol{\alpha}$ & ROS production (au/s/mg TC) \\
\hline$n C_{60}$ in the bulk & $0.52 \pm 0.09$ & $5.4 \pm 0.3$ \\
$n C_{60}<800 \mathrm{~nm}$ & $0.25 \pm 0.07(p<0.01)^{a}$ & $18.1 \pm 1.1(p<0.01)^{a}$ \\
$n C_{60}<450 \mathrm{~nm}$ & $0.08 \pm 0.04(p<0.01)^{a}$ & $31.5 \pm 1.4(p<0.01)^{a}$ \\
$n C_{60}<50 \mathrm{~nm}$ & $0.01 \pm 0.01(p<0.005)^{a}$ & $56.3 \pm 2.3(p<0.005)^{a}$ \\
\hline
\end{tabular}

${ }^{a}$ The $p$ value compared to the bulk aggregates.

VOL. 4 - NO. 9 - 5011-5018 - 2010 


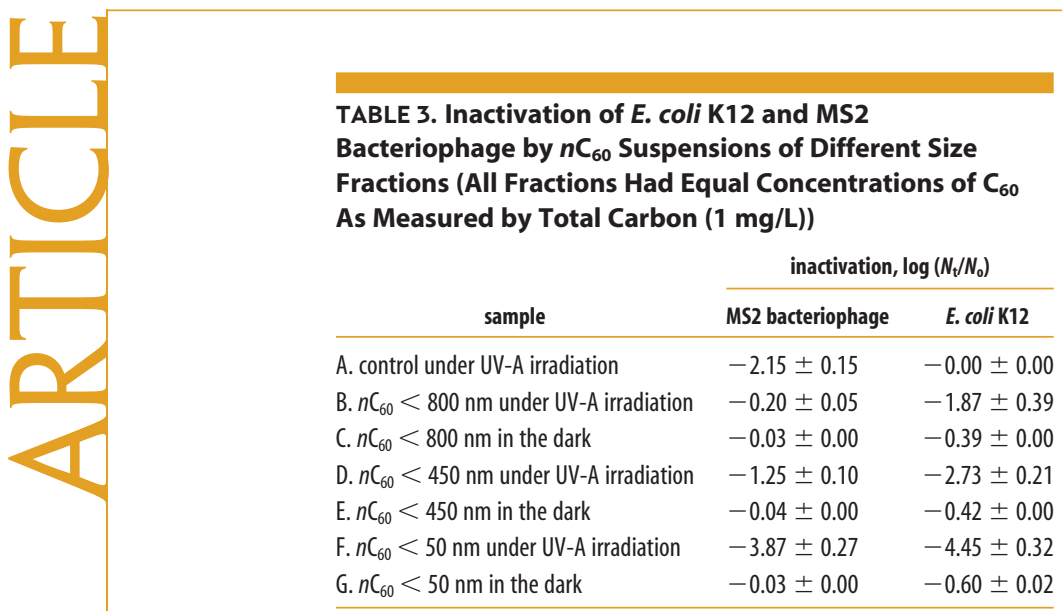

hydroxylation of the $\mathrm{C}_{60}$ with respect to ROS formation. Derivatization of the $\mathrm{C}_{60}$ cage reduces the quantum yield. The high degree of hydroxylation of fullerol is calculated to reduce the quantum yield of fullerol by an order of magnitude compared with underivatized $\mathrm{C}_{60}$. However, derivatization also enhances the affinity of the $C_{60}$ for the aqueous phase and results in an aggregate structure that enhances ROS generation due to reduced triplet-triplet interactions. Partial hydroxylation of the $C_{60}$ in the smallest size class may be adequate to alter size and structure of aggregates, but with less impact on the quantum yield of the $\mathrm{C}_{60}$.

ROS-Mediated Disinfection by the Fullerene Suspensions under Ultraviolet (UV) Irradiation. We have observed that conditions favoring ROS production by fullerol suspensions lead to inactivation of viruses., ${ }^{9,12}$ Thus, viral inactivation may also serve as an indicator of ROS generation. Table 3 shows the inactivation of Escherichia coli K12 and MS2 bacteriophage under the following conditions: (1) UV-A alone, (2) aqu/nC $60(<50,<450$, and $<800 \mathrm{~nm})+$ UV-A, and (3) aqu/nC 60 ( $<50,<450$, and $<800 \mathrm{~nm})+$ dark. A 60 min exposure to UV irradiation had negligible effect on the viability of $E$. coli $\mathrm{K} 12$, whereas significant inactivation was observed in the presence of UV-Asensitized aqu $/ \mathrm{nC}_{60}$. At the end of $1 \mathrm{~h}$ UV irradiation, $>4$ log inactivation of $E$. coli $\mathrm{K} 12$ occurred in the presence of aqu $/ \mathrm{nC}_{60}(<50 \mathrm{~nm}$ ) compared with approximately 2.5 log inactivation in the presence of larger aggregates. While MS2 inactivation did not occur in the dark, a small degree of inactivation of its host bacteria in the dark suggests that $\mathrm{C}_{60}$ may damage bacteria via a ROS-independent pathway, ${ }^{26}$ such as direct oxidation and that this latter pathway may be less sizedependent. Enhanced MS2 inactivation was observed in the presence of UV-sensitized aqu $/ \mathrm{nC}_{60}$ in the smallest fraction, achieving $>3.5 \mathrm{log}$ inactivation at the end of irradiation. Under UV irradiation, trends in E. coli K12 inactivation in the presence of $n C_{60}$ fractions paralleled those for MS2, with the highest inactivation occurring in the $<50 \mathrm{~nm}$ fraction. We speculate that the higher degrees of inactivation of $E$. coli K12 compared with MS2 are due to the presence of a large number of target sites on the bacteria, such as lipopolysacchairdes, glycoproteins, and various amino acid residues that are susceptible to damage by singlet oxygen. MS2 capsid proteins are largely composed of reactive amino acids (for example, histidine, tryptophan, and tyrosine), which are also excellent targets for singlet oxygen. It is interesting to note that MS2 inactivation by fractions larger than $50 \mathrm{~nm}$ was lower than that observed in the presence of UV alone. This apparent protective effect of particles on MS2 may result from a decreased exposure to UV, by hindered binding of MS2 with its host receptors, or possibly scavenging of UV-sensitized radicals by the $n \mathrm{C}_{60}$.

Transmission electron micrographs of E. coli K12 and MS2 bacteriophage following exposure to different size fractions of UV-sensitized aqu $/ n C_{60}$ for $60 \mathrm{~min}$ showed no visible damage in the cases of the bulk without membrane filtration (Figure 3a) and largest $(<800$ $\mathrm{nm}$ ) fraction (Figure $3 \mathrm{~b}$ ). However, aggregates exposed to the two smaller fractions ( $<450$ and $<50 \mathrm{~nm}$ ) showed compromised cell membrane integrity with less definition of the outer membrane, perhaps due to the release of cytoplasmic fluids (Figure 3c,d). Particles of $n \mathrm{C}_{60}$ were visible on the surface of the cell (Figure $3 c 1, c 2)$ and in the peptidoglycan layer and an opening of the cell membrane (see red arrows pointing the damaged portion Figure $3 \mathrm{~d} 1, \mathrm{~d} 2$ ). These observations are consistent with the inactivation results and singlet oxygen generation in the two $n C_{60}$ fractions smaller than $450 \mathrm{~nm}$. These images also highlight the possible role of fullerene proximity in delivering the singlet oxygen to biochemical moieties in the cell membrane and subsequent inactivation. The cells had a normal appearance in all samples where bacteria were exposed to $n C_{60}$ fractions in the dark (Figure $3 e$ ). Figure $3 \mathrm{f}-\mathrm{h}$ shows qualitative effects of UV alone and UV-sensitized aqu/ $n \mathrm{C}_{60}$ on the MS2 capsid architecture. In the dark (Figure 3f), MS2 has a near spherical appearance, while numerous irregularities appear following MS2 exposure to UV alone (Figure 3g). Capsids were severely damaged in the presence of aqu/ $n C_{60}$ after exposure to UV for 60 min (Figure $3 \mathrm{~h}$ ), and black circular spots are evident on several capsids, concurrent with high rates of inactivation $>3.5 \mathrm{log}$ in the presence of $<50 \mathrm{~nm}$ aqu $/ n \mathrm{C}_{60}$ aggregates. These same conditions produced the highest amount of singlet oxygen.

The hypothesized states of $n C_{60}$ present following consecutive filtration steps are summarized in Figure 4. At each filtration step, aggregates larger than membrane pore size are removed, selecting for an increasingly hydroxylated population of less dense aggregates, albeit at lower concentrations. This increasing hydroxylation is likely the reason for the decreases in the attachment coefficient and $K_{\mathrm{tw}}$ with decreasing aggregate size. Moreover, we speculate that hydroxylation, perhaps catalyzed by ROS production on the surface of the $n \mathrm{C}_{60}$, may lead to increasingly greater subdivision of 

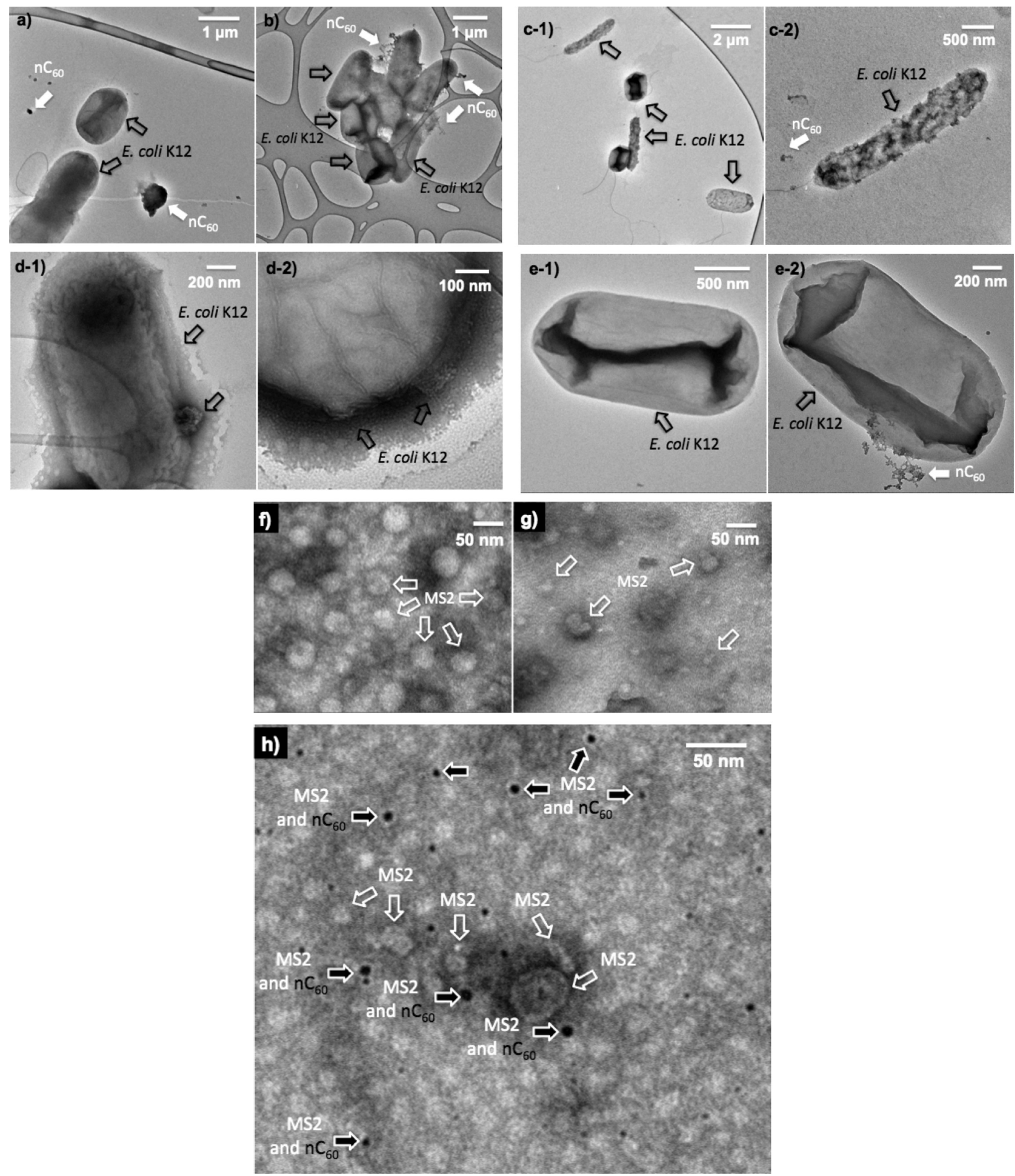

Figure 3. TEM images of $E$. coli $\mathrm{K} 12$ and MS2 bacteriophage in the fullerene suspensions. (a) $E$. coli $\mathrm{K} 12 \mathrm{in}$ aqu/nC 60 (bulk) after $1 \mathrm{~h} \mathrm{UV}$ irradiation. (b) $E$. coli $\mathrm{K} 12$ in aqu/nC 60 smaller than $800 \mathrm{~nm}$ after $1 \mathrm{~h}$ UV irradiation. (c1,c2) E. coli K12 in aqu/nC 60 smaller than $450 \mathrm{~nm}$ after $1 \mathrm{~h}$ UV irradiation. (d1,d2) E. coli K12 in aqu/nC 60 smaller than $50 \mathrm{~nm}$ after $1 \mathrm{~h} \mathrm{UV}$ irradiation. (e1,e2) E. coli K12 in aqu/nC $60 \mathrm{smaller}$ than $50 \mathrm{~nm}$ in the dark. (f) MS2 bacteriophage without $n C_{60}$ in the dark after $1 \mathrm{~h}$. (g) MS2 bacteriophage without $n C_{60}$ after $1 \mathrm{~h}$ UV irradiation. (h) MS2 bacteriophage in aqu/nC $\mathrm{C}_{60}$ smaller than $50 \mathrm{~nm}$ after $1 \mathrm{~h}$ UV irradiation. All fractions had equal concentrations of $\mathrm{C}_{60}$ as measured by total carbon ( $1 \mathrm{mg} / \mathrm{L}$ ).

the initial aggregates over time. This would result in an increase in the relative portion of these more reactive, small aggregates over time, which in turn would increase ROS production. By this mechanism, ROS production by the $n C_{60}$ dominates the self-quenching ef- fects (e.g., triplet-triplet annihilation) and results in the increase of net ROS generation and greater microbial toxicity.

In summary, suspensions of $\mathrm{nC}_{60}$ are far from homogeneous. Parameters obtained by averaging over the 


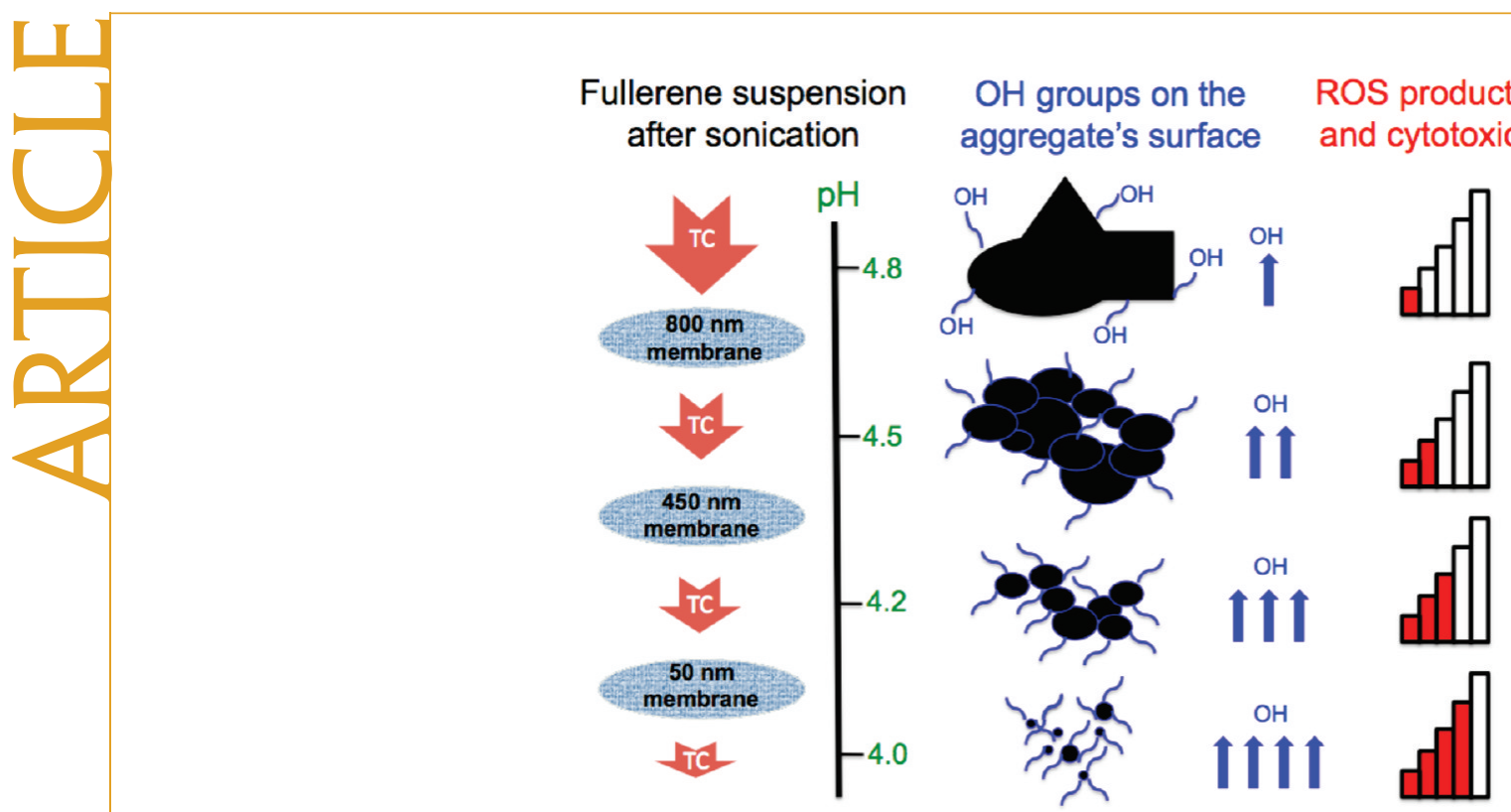

Figure 4. Schematic of the proposed mechanism for fullerene size-dependent effects on ROS production and microbial toxicity.

mass of material in suspension, or those where large aggregates dominate measurement (e.g., hydrodynamic diameter determined by DLS), may not be accurate descriptors of the behavior of these suspensions. Smaller $\mathrm{C}_{60}$ aggregates are hydroxylated to a greater degree, imparting these aggregates with a greater affinity for the aqueous phase, lower deposition rates, and higher potentials for ROS production per mass of fullerene. These differences translate into substantially greater photosensitized toxicity of small fullerene aggregates to bacteria and viruses. Thus, the performance and interpretation of experiments evaluating fullerene transport and toxicity must take into account the heterogeneity of these suspensions.

\section{METHODS}

Preparation and Characterization of Colloidal Fullerene Suspensions. Fullerene $\left(C_{60}, 99.9 \%\right)$ and hydroxylated fullerene (fullerol, $\mathrm{C}_{60}(\mathrm{OH})_{24}$ ) were purchased from MER (Tucson, AZ). A colloidal suspension of fullerene (aqu/nC $\mathrm{C}_{60}$ ) was prepared using a sonication method without any organic solvent. ${ }^{10} \mathrm{~A}$ total of $100 \mathrm{mg}$ of $\mathrm{C}_{60}$ powder was added to $200 \mathrm{~mL}$ of ultrapure water (resistivity $>18.2 \mathrm{M} \Omega \cdot \mathrm{cm}$ and dissolved organic carbon concentration $<30$ $\mu \mathrm{g} / \mathrm{L})$ in a beaker. The mixture was then subjected to sonication (20 min (pulse on)/10 min (pulse off)) with a high-energy probe (S-4000, Misonix, Qsonica, LLC, Newtown, CT) for $15 \mathrm{~h}$ in the dark (applied energy was approximately $4.3 \mathrm{MJ}$ ). The resultant suspension was fractionated by successive filtration with membranes having nominal pore sizes of $800 \mathrm{~nm}$ (Nylaflo, Pall Life Sciences, Port Washington, NY), $450 \mathrm{~nm}$ (Nylaflo, Pall Life Sciences, Port Washington, NY), and $50 \mathrm{~nm}$ (Isopore, Millipore, Billerica, MA). Each membrane was rinsed with $200 \mathrm{~mL}$ of ultrapure water before use. Stock fullerol $\left(\mathrm{C}_{60}(\mathrm{OH})_{24}\right)$ suspensions were prepared by adding fullerol in powder form to ultrapure water as described previously. ${ }^{10}$ Both fullerene and fullerol suspensions were stored in a refrigerator at $4{ }^{\circ} \mathrm{C}$ before use. Total carbon (TC) concentrations of the $\mathrm{C}_{60}$ and fullerol suspensions were measured by a total organic carbon (TOC) analyzer (TOC5050A, Shimadzu, Columbia, MD). Oxidation-reduction potential (ORP) and $\mathrm{pH}$ of the fullerene suspensions were measured using ORP (Orion 9678BNWP, Thermo Scientific) and pH electrodes with a benchtop pH meter (Accumet Excel XL20, Fisher Scientific, Pittsburgh, PA). Particle size distribution by DLS at a $173^{\circ}$ angle and $\zeta$-potential were measured by Zetasizer Nano (Malvern Instrument, Bedford, MA).

Reactive Oxygen Species Measurement. Commonly used procedures for measuring singlet oxygen and superoxide were employed here. All samples were tested in triplicate within two weeks after preparation. XTT (2,3-bis(2-methoxy-4-nitro-5-sulfophenyl)-2Htetrazolium-5-carboxanilide) was obtained from Sigma-Aldrich
(St. Louis, MO). Singlet oxygen sensor green (SOSG) was purchased from Molecular Probes-Invitrogen (Carlsbad, CA). ${ }^{30}$

Transmission Electron Microscopy. High-magnification images of the suspensions are obtained by TEM (FEI Tecnai G2 Twin, Hillsboro, OR). Ten microliters of each sample was dropped on a lacey carbon/Cu grid (300 mesh, Electron Microscopy Sciences, Hatfield, PA) and dried in air before TEM measurement. Image analysis of the TEM pictures obtained was performed by using an Image-Pro version 4.5 (Media Cybernetics, Inc. Bethesda, MD). MS2 bacteriophages were negatively stained with $2.5 \%$ phosphotungstic acid (Fluka, Milwaukee, WI) prior to imaging.

Toluene-Water Partition Experiments. A toluene - water partition coefficient $\left(K_{\mathrm{tw}}\right)$ was determined as the basis for quantifying the relative affinity of these materials for the aqueous phase. The ability to extract fullerene aggregates into toluene from an aqueous matrix was first evaluated. Briefly, $5 \mathrm{~mL}$ of each suspension ( $\mathrm{TC}=5 \mathrm{mg} / \mathrm{L}$ ) was placed in a glass vial containing $5 \mathrm{~mL}$ of ultrapure water with $20 \mathrm{mM} \mathrm{KCl}$ prior to the addition of $10 \mathrm{~mL}$ of toluene. Preliminary experiments optimized the concentration of $\mathrm{KCl}$, at which the extract of $n \mathrm{C}_{60}$ was enhanced, and volume ratio between water and toluene (data not shown). Then, the vial was vertically mixed overnight, and the supernatant containing the fullerene was drawn for further analysis. Last, quantitative analysis of fullerene was performed using a high-performance liquid chromatograph (HPLC) (ProStar, Varian, Palo Alto, CA) equipped with a Cosmosil Buckyprep column (Nacalai USA, Inc., San Diego, CA). The chromatographic separation was performed in isocratic mode at a constant flow rate of $1 \mathrm{~mL} / \mathrm{min}$ with a mobile phase of $100 \%$ toluene at $285 \mathrm{~nm}$ UV wavelength. The $K_{\mathrm{tw}}$ was calculated by the following equation.

$$
K_{\mathrm{tw}}=\frac{C_{\mathrm{t}}}{C_{\mathrm{w}}}
$$


where $C_{t}$ is the concentration of fullerene in toluene and $C_{w}$ is the concentration of fullerene in water (TC $=5 \mathrm{mg} / \mathrm{L}$ ).

Column Experiments. Transport of $n C_{60}$ through porous media was evaluated to quantify the relative affinity of each fraction of these fullerenes for the medium. A chromatography column (10 mm o.d. $\times 40 \mathrm{~mm} \mathrm{~L}$, GE Health Care, Piscataway, NJ) packed with spherical glass beads having nominal diameter of approximately $360 \mu \mathrm{m}$ was used for this purpose. The background solution in this study was ultrapure water driven by a peristaltic pump (Cole-Parmer Instrument Company, Chicago, IL) at a flow rate of $0.96 \mathrm{~mL} / \mathrm{min}$ as compared to the flow rate of $0.04 \mathrm{~mL} / \mathrm{min}$ of the fullerene suspension driven by a syringe pump (Harvard Apparatus, Holliston, MA). To quantify the relative fullerene concentration, the UV absorbance of fullerene was continuously measured by UV/vis spectrophotometer (UV-vis 2810, Hitachi, Pleasanton, (A) at the wavelength of $198 \mathrm{~nm}$, which is the maximum absorbance peak of the spectrum $(198,285$, and $334 \mathrm{~nm})$. The influent concentration $\left(C_{0}\right)$ was measured without connecting the column, while the effluent concentration $(C)$ was measured for the suspension at the outlet from the column. The attachment efficiency $(\alpha)$ was then calculated as $^{31}$

$$
\alpha=\frac{2 d_{c}}{3 \eta_{0} L(\varepsilon-1)} \ln \left(\frac{C}{C_{0}}\right)
$$

where $\eta_{0}$ is the single-collector efficiency obtainable by using a correlation equation developed based on the numerical solution of the convective-diffusion equation governing the transport phenomena in the column used, ${ }^{32} \varepsilon$ is the porosity of the porous media, $d_{\mathrm{c}}$ the diameter of the collector, and $L$ the length of the column. The attachment efficiency $(\alpha)$ indicates the probability of attachment of a particle onto a collector surface (glass bead) per collision.

Image Analysis for Two-Dimensional Fractal Dimension $\left(D_{2}\right)$ of Fullerene Aggregates. The surface density of primary particles in a 2-D object with a fractal dimension $D_{2}$ is given by ${ }^{33}$

$$
\rho(d) \propto d_{0}^{-D_{2}} \times d_{\mathrm{T}}^{D_{2}-2}
$$

where $d_{0}$ is the characteristic size of the primary particles and $d_{T}$ is the mean diameter of aggregates determined from TEM images. Defining this surface density as the surface area of particles contained in the smallest circle containing the aggregate, we can also write

$$
\rho(d)=\frac{N_{0} d_{0}^{2}}{d_{\mathrm{T}}^{2}}
$$

where $N_{0}$ is the number of primary particles in the aggregate.

Considering a negligible amount of particle overlap, we express the total area of the projected aggregate and then combine it with eqs 3 and 4 as

$$
A_{\mathrm{p}, \mathrm{tot}}=N_{0} \frac{\pi d_{0}^{2}}{4} \propto \frac{\pi}{4}\left(\frac{d_{\mathrm{T}}}{d_{0}}\right)^{D_{2}}
$$

which gives

$$
\log _{10} A_{\mathrm{p}, \mathrm{tot}}=D_{2} \log _{10} d_{\mathrm{T}}+B
$$

A plot of $A_{p, t o t}$ as a function of $d$ that is determined by TEM on log axes should therefore yield the 2-D fractal dimension $\left(D_{2}\right)$ of the projected aggregate, where $D_{2}$ and $B$ are a slope and an intercept of the log-log plot.

Detection of Reactive Oxygen Species from the Fullerene Suspensions. SOSG was used to measure singlet oxygen $\left({ }^{1} \mathrm{O}_{2}\right)$ concentrations in the fullerene suspensions. ${ }^{30}$ The fluorescence units (FSU) are sample measurements compared against a control sample in the dark used as a background (Modulus Single Tube 9200, Turner Biosystems, Sunnyvale, CA). XTT reduction was employed to measure the production of superoxide $\left(\mathrm{O}_{2}{ }^{--}\right)$. The reduction of $\mathrm{XTT}$ results in an increase in optical density at $470 \mathrm{~nm}$ that can be used to quantify the relative amount of superoxide present..$^{34,35}$
To study ROS production by the fullerene suspensions, experiments were performed using $10 \mathrm{~mL}$ sample in a glass beaker $(90$ $\mathrm{mm}$ o.d. $\times 115 \mathrm{~mm}, \mathrm{H}$ ) with a water jacket connected to a recirculating water chiller for temperature control and submitted to UV-A irradiation as described in the previous study..$^{10}$ All experiments were performed in triplicate. Student's $t$ test was used to assess the significance of the results employing a $95 \%$ confidence interval.

Evaluation of Bacterial and Viral Activity in the Fullerene Suspensions. MS2 bacteriophage (American Type Culture Collection (ATCC) 15597-B1) and E. coli K12 (ATCC 25404) were used as model virus and bacteria, respectively, to investigate the size-dependent toxic effects of aqueous $n C_{60}$. In the experiments with viruses, pure virus stock suspension free of bacterial debris and media residue was obtained using the following procedure: First, MS2 was cultured on double-layer agar plate with E. coli (ATCC 15597) as the host incubated at $37^{\circ} \mathrm{C}$. Next, the phages on the top agar were extracted into $1 \times$ phosphate buffered saline (PBS, $\mathrm{pH} 7.3$ ) and precipitated using $10 \%$ polyethylene glycol (PEG 6000 , Sigma Aldrich) at $4^{\circ} \mathrm{C}$ for $1 \mathrm{~h}$. The precipitated phages were centrifuged at $10000 \mathrm{~g}$ for $15 \mathrm{~min}$ at $4{ }^{\circ} \mathrm{C}$, and the supernatant was decanted and the pellet resuspended in PBS $(1-2 \mathrm{~mL}$ per $100 \mathrm{~mL}$ ) by overnight incubation at $4^{\circ} \mathrm{C}$. Further, the residual bacterial debris and polyethylene glycol (PEG 6000) in the phage suspension were removed by centrifugation at $5000 \mathrm{~g}$ for 10 min at $4{ }^{\circ} \mathrm{C}$ and chloroform (1:1 sample-to-chloroform), respectively. The phage containing aqueous phase was separated from white organic phase by centrifugation at $5000 \mathrm{~g}$ for $10 \mathrm{~min}$ at 4 ${ }^{\circ} \mathrm{C}$. The suspension was furthermore concentrated by ultracentrifugation at $103000 \mathrm{~g}$ for $2.5 \mathrm{~h}$ at $12{ }^{\circ} \mathrm{C}$, and the phage pellet was resuspended in PBS. The viruses were counted in terms of plaque forming units (PFU) that are between 30 and 150 per plate. This procedure yielded an initial stock concentration of $1.1 \times 10^{12} \mathrm{PFU} / \mathrm{mL}$, where PFU is plaque-forming unit. The target concentration of phages in all of the test solutions is $1-1.2$ $\times 10^{6} \mathrm{PFU} / \mathrm{mL}$. Exponential phase $E$. coli $\mathrm{K} 12$ grown in tryptone broth at $37^{\circ} \mathrm{C}$ were harvested by centrifugation at $5000 \mathrm{~g}$ for 10 min at $4{ }^{\circ} \mathrm{C}$ and resuspended in PBS to obtain a stock suspension. The initial concentration of bacteria in all test solutions is $1-1.5$ $\times 10^{6} \mathrm{CFU} / \mathrm{mL}$. Only $30-150 \mathrm{CFU}$ (colony-forming unit) from each plate were used for counting. The test solutions consisted of the following: (1) MS2 or E. coli K12 in $1.6 \mathrm{mM}$ PBS (pH 7.3 adjusted) exposed to UV-A, (2) MS2 or E. coli K12 $+n C_{60}$ in 1.6 $\mathrm{mM}$ PBS exposed to UV-A, and (3) MS2 or E. coli $\mathrm{K} 12+n \mathrm{C}_{60}$ in $1.6 \mathrm{mM}$ PBS kept in the dark. Inactivation of MS2 and E. coli K12 by the fullerene aggregates was calculated by the following equation.

$$
\text { Inactivation }=\log \left(\frac{N_{\mathrm{t}}}{N_{0}}\right)
$$

where $N_{\mathrm{t}}$ is the number of MS2 or $E$. coli $\mathrm{K} 12$ per mL after 60 min and $N_{0}$ is the initial number of MS2 or E. coli K12 per mL at time 0 min.

Acknowledgment. This material is based upon work supported by the National Science Foundation (NSF) and the Environmental Protection Agency (EPA) under NSF Cooperative Agreement EF-0830093, Center for the Environmental Implications of NanoTechnology (CEINT). Any opinions, findings, conclusions or recommendations expressed in this material are those of the author(s) and do not necessarily reflect the views of the NSF or the EPA. This work has not been subjected to EPA review and no official endorsement should be inferred.

Note Added after ASAP Publication: This paper was published August 13, 2010 with incomplete corrections in the "Characteristics of the Fullerene Suspensions" section. The revised version was published on August 18, 2010.

Supporting Information Available: Additional experimental details. This material is available free of charge via the Internet at http://pubs.acs.org. 


\section{REFERENCES AND NOTES}

1. Hoffman, M.; Hotze, E. M.; Wiesner, M. R. Environmental Nanotechnology: Applications and Impacts of Nanomaterials; McGraw-Hill: New York, 2007; pp 185-197.

2. Hotze, E. M.; Bottero, J.-Y.; Wiesner, M. R. Theoretical Framework for Nanoparticle Reactivity as a Function of Aggregation State. Langmuir 2010, 26, 11170-11175.

3. Lead, J. R.; Smith, E. Environmental and Human Impacts of Nanotechnology; Wiley-Blackwell: Chichester, UK, 2009.

4. Colvin, V. L. The Potential Environmental Impact of Engineered Nanomaterials. Nat. Biotechnol. 2003, 21, 1166-1170.

5. Nowack, B.; Bucheli, T. D. Occurrence, Behavior and Effects of Nanoparticles in the Environment. Environ. Pollut. 2007, 150, 5-22.

6. Nakanishi, I.; Fukuzumi, S.; Konishi, T.; Ohkubo, K.; Fujitsuka, M.; Ito, O.; Miyata, N. DNA Cleavage via Superoxide Anion Formed in Photoinduced Electron Transfer from NADH to Gamma-Cyclodextrin-Bicapped C60 in an Oxygen-Saturated Aqueous Solution. J. Phys. Chem. B 2002, 106, 2372-2380.

7. Orfanopoulos, M.; Kambourakis, S. Chmical Evidence of Singlet Oxygen Production from C-60 and C-70 in Aqueous and Other Polar Media. Tetrahedron Lett. 1995, 36, 435-438.

8. Yamakoshi, Y.; Sueyoshi, S.; Fukuhara, K.; Miyata, N. $\bullet \mathrm{OH}$ and $\mathrm{O}_{2}^{\bullet-}$ - Generation in Aqueous $\mathrm{C}_{60}$ and $\mathrm{C}_{70}$ Solutions by Photoirradiation: An EPR Study. J. Am. Chem. Soc. 1998, 120, 12363-12364.

9. Hotze, E. M.; Labille, J.; Alvarez, P.; Wiesner, M. R. Mechanisms of Photochemistry and Reactive Oxygen Production by Fullerene Suspensions in Water. Environ. Sci. Technol. 2008, 42, 4175-4180.

10. Chae, S. R.; Hotze, E. M.; Wiesner, M. R. Evaluation of the Oxidation of Organic Compounds by Aqueous Suspensions of Photosensitized Hydroxylated-C-60 Fullerene Aggregates. Environ. Sci. Technol. 2009, 43, 6208-6213.

11. Chae, S. R.; Wang, S. Y.; Hendren, Z. D.; Wiesner, M. R.; Watanabe, Y.; Gunsch, C. K. Effects of Fullerene Nanoparticles on Escherichia coli K12 Respiratory Activity in Aqueous Suspension and Potential Use for Membrane Biofouling Control. J. Membr. Sci. 2009, 329, 68-74.

12. Badireddy, A. R.; Hotze, E. M.; Chellam, S.; Alvarez, P.; Wiesner, M. R. Inactivation of Bacteriophages via Photosensitization of Fullerol Nanoparticles. Environ. Sci. Technol. 2007, 41, 6627-6632.

13. Kasermann, F.; Kempf, C. Photodynamic Inactivation of Enveloped Viruses by Buckminsterfullerene. Antiviral Res. 1997, 34, 65-70.

14. Sayes, C. M.; Gobin, A. M.; Ausman, K. D.; Mendez, J.; West, J. L.; Colvin, V. L. Nano-C-60 Cytotoxicity Is Due to Lipid Peroxidation. Biomaterials 2005, 26, 7587-7595.

15. Kai, Y.; Komazawa, Y.; Miyajima, A.; Miyata, N.; Yamakoshi, Y. [60]Fullerene as a Novel Photoinduced Antibiotic. Fullerenes, Nanotubes, Carbon Nanostruct. 2003, 11, 79-87.

16. Heymann, D. Solubility of Fullerenes C-60 and C-70 in Seven Normal Alcohols and Their Deduced Solubility in Water. Fullerene Sci. Technol. 1996, 4, 509-515.

17. Brant, J. A.; Labille, J.; Bottero, J.-Y.; Wiesener, M. R. Characterizing the Impact of Preparation Method on Fullerene Cluster Structure and Chemistry. Langmuir 2006 22, 3878-3885.

18. Kovochich, M.; Espinasse, B.; Auffan, M.; Hotze, E. M.; Wessel, L.; Xia, T.; Nel, A. E.; Wiesner, M. R. Comparative Toxicity of C60 Aggregates towards Mammalian Cells: Role of the Tetrahydrofuran (THF) Decomposition. Environ. Sci. Technol. 2009, 43, 6378-6384.

19. Labille, J.; Masion, A.; Ziarelli, F.; Rose, J.; Brant, J.; Villieras, F.; Pelletier, M.; Borschneck, D.; Wiesner, M. R.; Bottero, J. Y. Hydration and Dispersion of C-60 in Aqueous Systems: The Nature of Water-Fullerene Interactions. Langmuir 2009, 25, 11232-11235.

20. Lee, J.; Fortner, J. D.; Hughes, J. B.; Kim, J. H. Photochemical Production of Reactive Oxygen Species by C-60 in the
Aqueous Phase during UV Irradiation. Environ. Sci. Technol. 2007, 41, 2529-2535.

21. Lee, J.; Yamakoshi, Y.; Hughes, J. B.; Kim, J. H. Mechanism of C-60 Photoreactivity in Water: Fate of Triplet State and Radical Anion and Production of Reactive Oxygen Species. Environ. Sci. Technol. 2008, 42, 3459-3464.

22. Kratschmer, W.; Lamb, L. D.; Fostiropoulos, K.; Huffman, D. R. Solid C-60-A New Form of Carbon. Nature 1990, 347, 354-358.

23. Pickering, K. D.; Wiesner, M. R. Fullerol-Sensitized Production of Reactive Oxygen Species in Aqueous Solution. Environ. Sci. Technol. 2005, 39, 1359-1365.

24. Fortner, J. D.; Lyon, D. Y.; Sayes, C. M.; Boyd, A. M.; Falkner, J. C.; Hotze, E. M.; Alemany, L. B.; Tao, Y. J.; Guo, W.; Ausman, K. D.; et al. C-60 in Water: Nanocrystal Formation and Microbial Response. Environ. Sci. Technol. 2005, 39, 4307-4316.

25. Sayes, C. M.; Fortner, J. D.; Guo, W.; Lyon, D.; Boyd, A. M.; Ausman, K. D.; Tao, Y. J.; Sitharaman, B.; Wilson, L. J.; Hughes, J. B.; et al. The Differential Cytotoxicity of WaterSoluble Fullerenes. Nano Lett. 2004, 4, 1881-1887.

26. Lyon, D. Y.; Brunet, L.; Hinkal, G. W.; Wiesner, M. R.; Alvarez, P. J. J. Antibacterial Activity of Fullerene Water Suspensions $(n C(60))$ Is Not Due to ROS-Mediated Damage. Nano Lett. 2008, 8, 1539-1543.

27. Derjaguin, B. A Theory of Interaction of Particles in Presence of Electric Double Layers and the Stability of Lyophobe Colloids and Disperse Systems. Acta Physicochim. Urs. 1939, 10, 333-346.

28. Verwey, E. J. W. Theory of the Stability of Lyophobic Colloids. J. Phys. Colloid. Chem. 1947, 51, 631-636.

29. Markovic, Z.; Todorovic-Markovic, B.; Kleut, D.; Nikolic, N.; Vranjes-Djuric, S.; Misirkic, M.; Vucicevic, L.; Janjetovic, K.; Isakovic, A.; Harhaji, L.; et al. The Mechanism of CellDamaging Reactive Oxygen Generation by Colloidal Fullerenes. Biomaterials 2007, 28, 5437-5448.

30. Flors, C.; Fryer, M. J.; Waring, J.; Reeder, B.; Bechtold, U.; Mullineaux, P. M.; Nonell, S.; Wilson, M. T.; Baker, N. R. Imaging the Production of Singlet Oxygen In Vivo Using a New Fluorescent Sensor, Singlet Oxygen Sensor Green. J. Exp. Bot. 2006, 57, 1725-1734.

31. Yao, K. M.; Habibian, M. M.; Omelia, C. R. Water and Wastewater Filtration-Concepts and Applications Environ. Sci. Technol. 1971, 5, 1105-1112.

32. Tufenkji, N.; Elimelech, M. Correlation Equation for Predicting Single-Collector Efficiency in Physicochemical Filtration in Saturated Porous Media. Environ. Sci. Technol. 2004, 38, 529-536.

33. Feder, J. Fractals; Plenum Press: New York, 1988.

34. Ukeda, H. H.; Maeda, S. S.; Ishii, T. T.; Sawamura, M. M. Spectrophotometric Assay for Superoxide Dismutase Based on Tetrazolium Salt 3' -1-(Phenylamino)carbonyl-3,4tetrazoliumbis(4-methoxy-6-nitro)benzenesulfonic Acid Hydrate Reduction by Xanthine-Xanthine Oxidase. Anal. Biochem. 1997, 251, 206-209.

35. Bartosz, G. Use of Spectroscopic Probes for Detection of Reactive Oxygen Species. Clin. Chim. Acta 2006, 368 , 53-76. 